\title{
THE NEP ROSAT SURVEY
}

\author{
C.R. MULLIS, I.M. GIOIA ${ }^{1}$ AND J.P. HENRY \\ Institute for Astronomy, University of Hawaii \\ 2680 Woodlawn Drive, Honolulu, HI 96822, USA \\ ${ }^{1}$ Istituto di Radioastronomia del CNR \\ Via Gobetti 101, 40129, Bologna, ITALY
}

\section{Introduction}

The Rosat All-Sky Survey (Trümper 1991, Adv. Spce Res., 2, 241) has its largest exposure times, approaching $10 \mathrm{ks}$, at the ecliptic poles where the scan circles overlap. The North Ecliptic Pole (NEP) region covers a $9^{\circ} \times 9^{\circ}$ field, and contains a total of $465 \mathrm{X}$-ray sources detected at $>4 \sigma$ in the $0.1-2.4 \mathrm{keV}$. We are identifying all sources in the field. The principal derivative is a statistically complete sample of galaxy clusters appropriate for more fully characterizing X-ray Luminosity Function (XLF) evolution. We report preliminary results for two subregions which are identified to the $95 \%$ level. These subregions are observed to approximately the median NEP survey exposure. They enclose $16 \%$ of the survey area and contain $96 \mathrm{X}$-ray sources representing $21 \%$ of the total NEP. The typical mix of sources is consistent with the Einstein Extended Medium Sensisitivity Survey (EMSS; Gioia et al., 1990a, ApJS, 72, 567). In particular there are $56 \%$ AGN/ELG (59\% in the EMSS), $25 \%$ stars (27\% in the EMSS) and $14 \%$ clusters against $13 \%$ clusters in the EMSS.

\section{The Sample}

There are 13 clusters in the sample drawn from the completely identified subregions of the NEP. The observed $0.1-2.4 \mathrm{keV}$ count rates are converted to $0.5-2.0 \mathrm{keV}$ band fluxes using a $6 \mathrm{keV}$ thermal bremmstrahlung model including Galactic absorption and K-corrections. Compensations for flux falling outside the detect cell are based on the convolution of the RASS PSF (De Grandi private communication) and a King profile. 


\section{Evolution}

$\mathrm{X}$-ray luminosities of clusters span three orders of magnitude from $10^{42}$ to $10^{45} \mathrm{erg} \mathrm{s}^{-1}$, and any study of their statistical properties must take this into account. In the context of cluster evolution, the relevant quantity is the X-ray luminosity function. Gioia et al. (1990a, ApJ, 365, L35) and Henry et al. (1992, ApJ, 386, 408) analyzed the XLF for the EMSS cluster sample at $\mathrm{z} \sim 0.17$ and $\mathrm{z} \sim 0.33$. Their analysis suggests a deficit of high luminosity clusters at high redshifts by a factor of three. We reproduce the EMSS XLFs in Figure 1. Two models XLFs are overplotted. H92 from Henry et al. (1992) is the best-fitting theoretical function for the EMSS data. BCS is the recent determination of the low redshift XLF from the ROSAT Brightest Cluster Sample (Ebeling et al. 1997, ApJ, 479, L101). As a first step towards addressing the evolution issue, we add two preliminary NEP XLF determinations at high redshift. To date, $59 \%$ of the ROSAT NEP targets have been identified. The complete survey will yield roughly 65 clusters, comparable in size to the EMSS sample. This will provide a significant and unique extension to previous attempts to confirm evolution.

This work received partial support froin NSF grant AST95-00515 and NASA grants NAG5-2594 and NAG 5-2914.

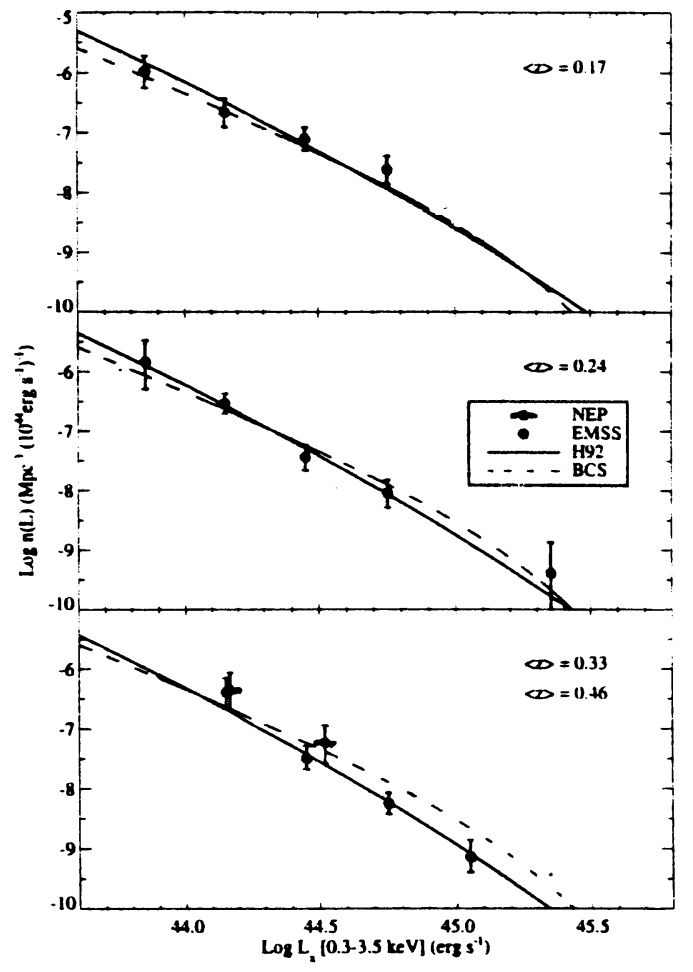

Figure 1. The XLF for clusters of galaxies in redshift shells. 